\title{
EIGENHEARTS FOR DIAGNOSIS OF CONGESTIVE HEART FAILURE (CHF)
}

\author{
V. Ahanathapillai ${ }^{1}$ and D.J. Hamilton ${ }^{1}$ \\ ${ }^{1}$ Department of Electronics and Electrical Engineering, University of Strathelyde, Glasgow, Scotland \\ vijideee.strath.ac.uk, david@eee.strath.ac.uk
}

\begin{abstract}
Common cardiac diseases such as cardiomyopathy, coronary artery diseases, and valve diseases, result in abnormal myocardial movement, which could eventually lead to heart failure, also called congestive heart failure (CHF). $\mathrm{CHF}$ is a disease in which the heart's ability to pump blood efficiently is lost. Possible presence of this disease and location of the abnormal activity can be diagnosed from patient's scan images, by determining the wall motion abnormalities. In this paper, a new principal component analysis (PCA) technique, Eigenhearts, is presented to diagnose the abnormal contractility of heart wall. Experiments were carried out using a preliminary set of simulated scan data and the results are discussed.
\end{abstract}

Keywords: Eigenhearts, Principal Component Analysis (PCA), Congestive Heart Failure (CHF), Echocardiography.

\section{INTRODUCTION}

Heart failure is a worldwide problem with high morbidity and mortality rates [1]. Congestive heart failure (CHF) is a state in which the heart becomes less efficient to pump blood. CHF has different forms such as systolic, diastolic, left-sided and right-sided heart failure [2]. Systolic heart failure is the most common type, caused by a failure in the contraction of heart muscles. This failure in contraction of the heart wall can be identified using various cardiac imaging data.

Principal component analysis (PCA) is a statistical technique that is widely used for pattern identification in large datasets with its main applications in fields such as face recognition (eigenfaces) and image compression [3, 4]. Recently, the PCA techniques have been used in the contour detection of the heart images $[5,6,7]$ and for lungs outline reconstruction [8]. In this paper, a technique to diagnose the contraction failure of heart muscle is presented using a PCA technique, referred hereafter as Eigenhearts.

Eigenhearts are obtained from echocardiography (echo) images, which is chosen over the other cardiac imaging techniques (MRI, CT). Echocardiography has the following advantages: reliable; harmless; less expensive method; and suitable for determining valve disease, left ventricle (LV) wall thickness, and the regional wall motion abnormalities [1].

\section{METHODOLOGY}

Eigenhearts, a PCA technique applied to the heart echo images, is one of the simple methods that work efficiently for pattern recognition problem. In this technique, the eigenvalues and eigenvectors of the covariance matrix for the echo image set is computed. Any heart image could then be represented as a weighted sum of these eigenvectors in a multidimensional heart space. To recognize a new heart image, the image should be within the heart space and should be close to one of the images in the image set. The mathematical derivation for the calculation of the eigenhearts and classification of heart images is detailed in next two sections, respectively.

A set of patient's echocardiograph images is simulated. The heart position at the systole and diastole phase is significant to get information on the contractility of the heart wall. The difference image between systolic and diastolic postures of the simulated echo data is computed and stored in a database. The Eigenhearts technique is applied to this difference image database. When a new patient's ultrasound image is obtained, the difference image is calculated and the Euclidean distance between this new heart image and the heart space is determined. If there is a similar case in the database, the new image will be close to that image and a similar condition could be recognised, helping in locating the contraction failure of the heart muscle. Once the contraction failure is detected, the risk of getting CHF in the future can be diagnosed. The records of other heart images from the same heart class could also be located and used as physician's cross-reference. When a similar case is not present, the input image is classified as a new heart class and the database is updated with this image and its medical records for future reference.

\section{Calculating Eigenhearts from scan images}

The calculation of the eigenhearts from the scan images is similar to the calculation of the eigenfaces from a face set $[3,4]$. Firstly, a database is formed with a set of $M$ heart scan images, $\Gamma_{1}, \Gamma_{2}, \Gamma_{3} \ldots \Gamma_{M}$, consisting of both normal and abnormal cardiac movements. The eigenhearts are formed from these scan images, which then become the basis of the heart space. The average image of the $\mathrm{M}$ scan images is calculated by

$$
\Psi=\frac{1}{M} \sum_{n=1}^{M} \Gamma_{n}
$$


Then a difference vector between each heart image and the average image is obtained as shown below.

$$
\Phi_{i}=\Gamma_{i}-\Psi
$$

Where $i=1 \ldots M$

The covariance matrix of the image set, $C$ is calculated by

$$
C=\frac{1}{M} \sum_{n=1}^{M} \Phi_{n} \Phi_{n}^{T}=A A^{T}
$$

Where $A=\left[\Phi_{1} \Phi_{2} \ldots \Phi_{M}\right]$

Eigenhearts (which is a set of $\mathrm{M}$ orthonormal vectors, $u_{k}$ ) and their associated eigenvalues, $\lambda_{k}$ of the above covariance matrix $\mathrm{C}$ are calculated [3] to best illustrate the distribution of the data.

\section{Using Eigenhearts for classification of the heart}

The Eigenhearts calculated as shown in the previous section were arranged in order of significance, based on their eigenvalues. The heart images can be described and classified using the top $M^{\prime}$ Eigenhearts (more significant), while the less significant ones can be ignored to reduce computation. The less significant Eigenhearts typically represent noise or minor features within the images. The number of Eigenhearts $\left(M^{\prime}<M\right)$ is determined heuristically.

In order to classify a new heart image, $\Gamma$, it is first converted into its eigenheart components by

Where $k=1 \ldots M^{\prime}$

$$
\omega_{k}=u_{k}^{T}(\Gamma-\Psi)
$$

Then, the weight vector $\Omega^{T}=\left[\omega_{1}, \omega_{2} \ldots \omega_{M^{\prime}}\right]$ is formed which describes the input of each eigenheart in representing the new heart image.

To find if the new image is a heart, the distance between the mean adjusted image, $\Phi=\Gamma-\Psi$ and its projection onto the heart space, $\Phi_{h}=\sum_{i=1}^{M^{\prime}} \omega_{i} u_{i}$ is determined as shown below.

$$
\varepsilon^{2}=\left\|\left(\Phi-\Phi_{h}\right)\right\|^{2}
$$

Once the new image is recognised as a heart image, to find a predefined heart class, which is close to this new image, the Euclidian distance is calculated between the new image and each heart class. The closest heart image is the one that minimises the Euclidian distance.

$$
\varepsilon_{k}^{2}=\left\|\left(\Omega-\Omega_{k}\right)\right\|^{2}
$$

Where $\Omega$ is the weight vector of the new image and $\Omega_{k}$ is the weight vector of the $k$ th class. If the minimum Euclidian distance $\varepsilon_{k}$ is less than a selected threshold $(\theta)$, the new image is said to belong to the $k$ th class. Based on the new image's classification into a predefined heart class, the new image can be classified as normal or abnormal. If the new image cannot be classified into an existing heart class, it can be classified as a new class on its own or with additional clinical input, be used to enhance the database by extending on existing class- heart space to include it.

\section{EXPERIMENTS AND RESULTS}

Echo images can be recorded in different views, each of which is important to identify critical parameters to evaluate different heart conditions. Here, the short axis mid-cavity view is considered. The echo images are captured over a time period, to cover a complete cardiac cycle. An average of 20 frames is present in each echo movie. Here, when an echocardiogram of a patient is obtained, an end-systole frame and an end-diastole frame are extracted from the movie. Then, the difference image is obtained from the two frames, which would give information on endocardial and epicardial movement. Fig. 1 shows clear bands in a difference image from a patient where the inner white band represents the endocardial movement and the outer white band represents the epicardial movement. Endocardium is the innermost layer of heart, which is composed of endothelial tissue. The outer layer of the heart tissue is called the epicardium, which is the inner layer of pericardium. The difference image obtained from each patient, in this case simulated ones, is stored in a database.

\section{Database of heart images}

The experiments are carried out with a simulated database with an image resolution of $130 \times 123$ pixels, stored in tagged image file format (tiff file). The images are gray scale with pixel values ranging from 0 to 255. The database consists of 32 synthesized images of short axis mid-cavity echocardiograms representing 4 different classes with 8 images each. To locate the contraction failure abnormality, the myocardium and the left ventricular cavity are segmented. The myocardial segments are considered based on the standards [9], which suggest that the left ventricle should be divided into 17 segments. These 17 segments can be assigned

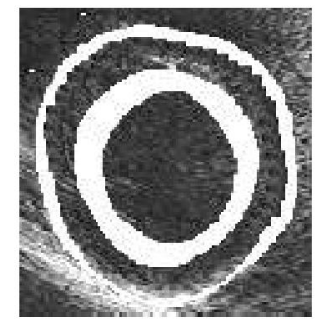

Fig. 1 Difference image showing two white bands - inner band represents the endocardial movement and the outer band represents the epicardial movement. 


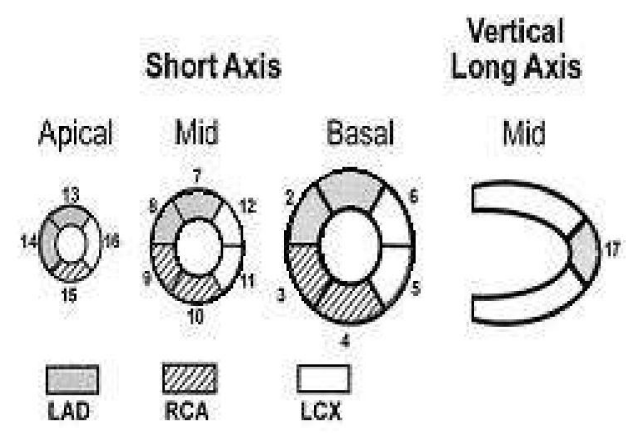

Fig. 2 Assignment of the 17 myocardial segments to the territories of the left anterior descending (LAD), right coronary artery (RCA), and the left circumflex coronary artery (LCX) [9].

to one of the three major coronary arteries, as shown in Fig 2. In the short axis view, the mid-cavity slice is divided into 6 segments of $60^{\circ}$ each, named mid anterior, mid anterioseptal, mid inferoseptal, mid inferior, mid inferolateral and mid anterolateral. Based on these standards, a database is formed with simulated data. The database consists of normal heart images and heart images with contraction failure in three (out of the six) different segments. Images in the database are grouped into different classes based on the segment abnormality present (same segment failure are grouped into a class). The classes described in here are: class1 - normal; class $2-7^{\text {th }}$ LV segment abnormality; class $3-9^{\text {th }}$ LV segment abnormality; class $4-11^{\text {th }}$ LV segment abnormality. When the new image is classified into one of the classes, 2, 3 or 4, the image is said to have a contraction failure in that particular segment. By locating the segment, the cause for the potential heart failure could be diagnosed. The fault in the corresponding coronary artery that supplies blood can be identified.

The first experiment is carried out to investigate the classification of a new image (not in the database) with a segment deformity into the correct class to locate the damaged segment. As the images in the database are limited, when one of the images is considered as a new image, it is removed from the database, also called the

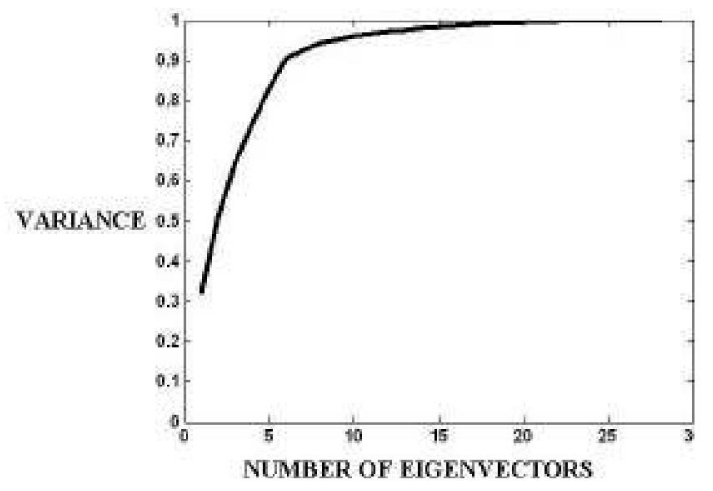

Fig. 3 Variance plot (28 image database)
(1)
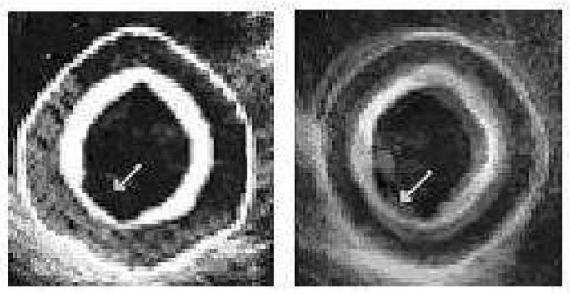

(2)
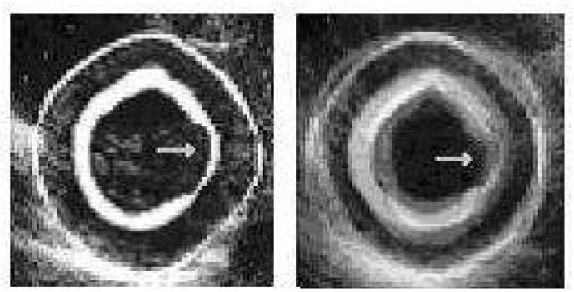

(3)
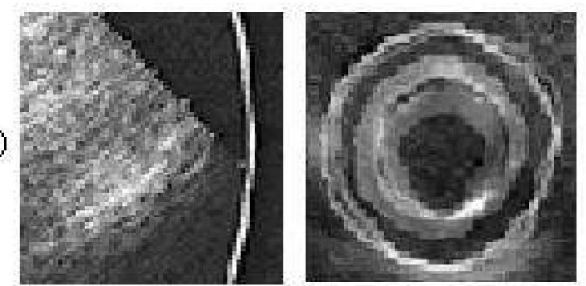

Fig. 4 Input images (left) and the reconstructed images (right). The arrows in the figure show the contraction failure in the segment. (1) Segment 11, (2) Segment 7, (3) Not a heart.

'leave one out method'. The variance between the images in the database is low and hence the number of top eigenvectors used for recognition must be high to give discrimination. Fig 3 shows the variance plot for the test performed with 28 images in the database for which $97 \%$ variance corresponds to top 12 eigenvectors. Correct recognition for $69 \%$ was obtained. Fig 4 shows few examples of the new images and their reconstructed images. When a new image could not be classified into one of the predefined classes, it can be classified as a new class.

An experiment was also performed with 20 and 24 images in the database. The percentage of correct classification obtained for these experiments were $56 \%$ and $62 \%$, respectively. From these results shown in Table 1 , it can be noted that the percentage of correct recognition increases with increase in the number of images in the database and in each class. Performing the experiment with a huge database with a large number of images in each class and choosing the accurate threshold values should improve the obtained result of $69 \%$.

In the second experiment, the effects of the severity of the segment contraction abnormality in recognising the segment are investigated. Modifications are made to all images other than the normal images in such a way that the abnormality is reduced representing an earlier stage of the abnormality. An example of the modified seg- 
ment abnormality is shown in Fig 5 . The aim of this experiment was to see if the correct segment is identified with the earlier stage deformity. 21 images were used for testing, out of which correct classification was obtained for 14 images. The correct recognition of $67 \%$ should also be improved by using a larger database.

\begin{tabular}{|c|c|c|}
\hline $\begin{array}{c}\text { Number of } \\
\text { images in the } \\
\text { database }\end{array}$ & $\begin{array}{c}\text { Number of im- } \\
\text { ages in each class }\end{array}$ & $\begin{array}{c}\% \text { of correct } \\
\text { recognition }\end{array}$ \\
\hline 20 & 5 & 56 \\
\hline 24 & 6 & 62 \\
\hline 28 & 7 & 69 \\
\hline
\end{tabular}

Table 1: Percentage of correct recognition obtained by increasing the number of images in the database.

The image background was changed and the correct recognition rate was observed. Correct recognition was obtained for all the three test images. This suggests that the background does not have a great effect as this technique considers the image as a whole for the recognition.

\section{CONCLUDING REMARKS}

Various image-processing techniques are used routinely for the diagnosis of the abnormal heart contraction from patient data. In this paper, a new eigenhearts technique is applied to the difference echo images to locate such contraction failures in the heart wall. The simulated experimental results show that this technique gives an overall detection rate of about $60 \%$, which could be improved by the making the database larger, and optimising thresholds. Dividing the difference images into 6 segments can extend this approach further and each segment could then be compared with the corresponding segment database to classify the level of severity. Once the contraction failure is diagnosed and located, the increased chance of the person developing a heart failure could be diagnosed, helping in early treatment for the condition. Also, the records of the patients with similar condition can readily be identified for cross-reference.
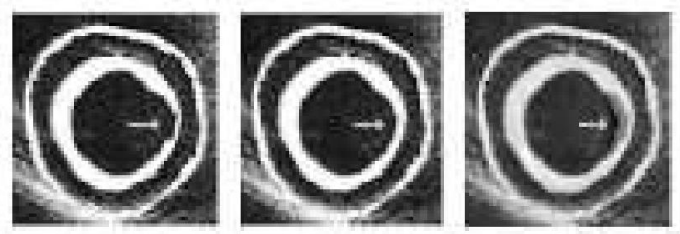

Fig. 5 Original image (left), modified image (middle) and the reconstructed images (right). The arrows in the figure show the contraction failure in the segment.

\section{REFERENCES}

[1] Zevitz M.E. (2005): 'Heart Failure', Emedicine, http://www.emedicine.com/med/topic3552.htm

[2] Bales A.C. and Sorrentino M.J. (1997): 'Causes of congestive heart failure: Prompt diagnosis may affect prognosis', Postgraduate Medicine symposium, 101(1).

[3] Turk M.A., Pentland A.P. (1991): 'Eigenfaces for recognition', Journal of Cognitive Neuroscience, 3, pp. 71-86.

[4] Turk M.A., Pentland A.P. (1991): 'Face recognition using eigenfaces', Proceedings of the IEEE Computer Society Conf. On Computer Vision and Pattern Recognition, pp. 586-591.

[5] Bosch J.G., Nijland F., Mitchell S.C., Lelieveldt B.P.F., Kamp O., Reiber J.H.C., Sonka M. (2005): 'Computer-aided Diagnosis via model-based shape analysis: Automated classification of wall motion abnormalities in echocardiograms', Academic Radiology, 12 (3), pp. 358-367.

[6] Cootes T.F., Hill A., Taylor C.J., Haslam J. (1994): 'The use of active shape models for locating structures in medical images', Image and Vision Computing, 12 (6), pp. 335-366.

[7] Maniam J., Narainasamy S. (2005): 'Search of Dynamic Magnetic Resonance images using active shape model', Sunway Academic Journal, 2, pp. $77-83$.

[8] Serpen G., Iyer R., Elsamaloty H.M.,Parsai E.I. (2003): 'Automated lung outline reconstruction in ventilation -perfusion scans using principal component analysis techniques', Computers in Biology and Medicine, 33, pp. 119-142.

[9] Cerqueira M.D., Weissman N.J., Dilsizian V., Jacobs A.K., Kaul S., Laskey W.K., Pennell D.J., Rumberger J.A., Ryan T., Verani M.S. (2002): 'Standardized myocardial segmentation and nomenclature for tomographic imaging of the heart', Circulation, 105, pp. 539 - 542. 